\title{
A Study on the Continuance Intention of O2O Fresh Agricultural Products E-Commerce*
}

\section{$\mathrm{O} 2 \mathrm{O}$ 를 활용한 신선한 농산품 전자상거래의 지속적 사용의도에 관한 연구}

\author{
Wei $\mathrm{GU}(\text { 고위) })^{* *}$, Peng $\mathrm{BAO}(\text { 포붕 })^{* * *}$, Jong-Ho LEE(이종호 $)^{* * * *}$
}

Received: September 03, 2019. Revised: September 18, 2019. Accepted: October 05, 2019.

\section{Abstract}

Purpose - This study focuses on the continuance intention of $\mathrm{O} 2 \mathrm{O}$ fresh agricultural products e-commerce. By literature review, this paper looks through the classical theories which are often applied to study use behavior and continuance intention on the electronic commerce area. Ultimately based on the expectancy theory, Technology acceptance model, success model as well as trust model, a model of the continuance intention of fresh products $\mathrm{O} 2 \mathrm{O}$ electronic commerce application is established.

Research design, data, and methodology - Among users Chinese consumers have been chosen who have use experience as the research objects. From October 2, 2018 to November 2, 2018, 685 questionnaires in total were collected by the online release and collection. Expect for the negative questionnaires, the remaining 650 pieces of data are statistically analyzed. The collected data were firstly be analyzed by SPSS Ver. 25 on its frequency, reliability and exploratory factors. Then AMOS Ver. 25 is applied to the Confirmatory Factor Analysis, Discriminant Validity and hypothesis testing of the Structural Equation Modeling. Finally, the following research conclusions could be obtained from the hypothesis testing.

Results - Firstly, in the extended IS model, quality factor for hypothesis testing, service quality, information quality and delivery quality have obviously present positive influences on satisfaction respectively. Secondly, in the hypothesis testing part of ECM-ISC model and UTAUT model, all hypotheses have presented accepted results. Especially from expectation confirmation to usefulness perception, the influence factor achieves 12.603, In the hypothesis of continuance intention, the influence factor of social influence on continuance intention is 7.748 and also it is the most remarkable one.

Conclusions - The results show that the service quality of $\mathrm{O} 2 \mathrm{O}$ fresh agricultural products e-commerce has the greatest impact on satisfaction, while the perceived usefulness of consumers has the most significant impact on $\mathrm{O} 2 \mathrm{O}$ fresh agricultural products for sustainable use intention. This thesis makes up for the blank of O2O fresh food e-commerce for sustainable use intentions, and provides a theoretical basis for consumers' sustainable use behavior, and practical enlightenment for the sustainable development of $\mathrm{O} 2 \mathrm{O}$ fresh agricultural products e-commerce.

Keywords: Fresh Agricultural Products, O2O, Expectation Confirmation, D\&M Model, ECM-ISC Model, Continuous Intention.

JEL Classification: M10, M16, M19.

* This is a partly abbreviated and modified article based on Doctoral thesis by Gu.

** First Author, Ph.D in Electronic Commerce, Kongju National University, Korea.

Tel: +82-41-850-8255, Email: earily@naver.com

*** Co-Author, Ph. D. Student, Dept. of Electronic Commerce, Kongju National University, Korea.

Tel: +82-41-850-8255, Email: baopeng0805@naver.com

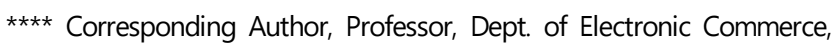
Kongju National University, Korea.

Tel: +82-41-850-8257, Email: leejh@kongju.ac.kr

(c) Copyright: Korean Distribution Science Association (KODISA)

This is an Open Access article distributed under the terms of the Creative Commons Attribution Non-Commercial License (https://creativecommons.org/licenses/by-nc/4.0/) which permits unrestricted non-commercial use, distribution, and reproduction in any medium, provided the original work is properly cited. 


\section{1. 서론}

최근 몇 년 동안 중국 국민경제의 발전과 함께 국민 실소득 이 지속해서 증가하였고, 특히 대도시 사람들의 생활수준이 더 높아지면서 중국 소비자들의 신선한 농산물과 우수한 서비스 에 대한 수요가 끊임없이 증가하였다. iResearch(2018)의 보고 서에 따르면 2017년 중국 신선식품의 시장규모는 17,897 억 위 안에 달하는 것으로 나타났고 2016년도 대비 $6.6 \%$ 증가하였 으며, 중국의 신선식품 전자상거래 시장의 거래 규모는 2017 년 약 1,391 억 위안으로 전년 대비 $134 \%$ 로 급속하게 증가하 였으며 2018년은 약 1,948억 위안으로 예상한다. 그러나 신선 식품은 쉽게 변질되고 유통기한이 비교적 짧으므로 제품의 신 선도가 매우 중요하고, 소비자들이 자주 구매하는 품목이기 때 문에 유통의 범위가 제한적이라는 특성을 갖는다(Yu \& Ren, 2018). 신선도를 유지하기 위해 일반적으로 소비자는 한 번에 많은 양의 상품을 구매하지 않는다. 이러한 신선식품 자체가 가진 고유한 상품의 특성은 $\mathrm{O} 2 \mathrm{O}$ 서비스 형태의 전자상거래에 적합하다고 볼 수 있다. $\mathrm{O} 2 \mathrm{O}$ 서비스는 일반적인 전자상거래의 가장 큰 단점인 비대면 거래와 긴 배송 기간이라는 한계점을 일부 극복할 수 있기 때문이다.

일반적으로 $\mathrm{O} 2 \mathrm{O}$ 신선식품 전자상거래는 크게 세 가지로 방식으로 구분되고, 가장 대표적인 $\mathrm{O} 2 \mathrm{O}$ 신선식품 전자상거래 의 방식은 한 지역에 자사의 영업점을 구축해 전치창고(前置 仓库)에서 소비자들이 앱을 통해 상품을 주문하고 대금을 결 제한 후, 상품을 배송받는 방식이다. 이러한 상품 배송은 $\mathrm{O} 2 \mathrm{O}$ 신선식품 전자상거래의 발전에 매우 중요한 요인을 볼 수 있 다. 특히 배송속도, 직원의 친절함 등의 요인은 배송 품질의 최적화와 고객 만족 제고에 큰 영향을 미친다(Oh \& Hyun, 2016; Gao \& Ko, 2017; Dai \& Lee, 2018). O2O 전자상거래 의 배송 서비스는 오프라인상의 서비스뿐만 아니라 온라인 주 문을 통해 직접 오프라인 매장을 방문하는 방식으로도 제공되 고 있다. 이렇게 소비자들의 직접 방문을 유도하는 방식은 실 제 오프라인 매장에서의 경험과 느낌을 제공하기 때문에 지속 적이고 장기적인 고객을 확보하는 데 긍정적인 영향을 미칠 수 있고, 배송 비용의 절감 효과도 얻을 수 있다.

소비자들은 전자상거래를 통해 신선식품을 구매할 때, 그들 이 선택하는 상품에 대한 기대와 효용 가치가 일치하기를 희 망한다. 그러나 기업 간의 가격 경쟁으로 인해 새로운 전자상 거래 플랫폼의 제품 및 서비스 품질 수준의 차이가 존재한다. 새로운 플랫폼에 대한 사용자들의 인식 부족과 함께 시스템과 정보의 품질에 차이가 있는 것이 현실이다.

특히 신선식품의 고유한 속성으로 인해 품질이나 서비스가 소비자의 기대에 부응하지 못하거나 소비자에게 불만스러운 쇼핑 경험은 지속적인 구매 의도에 치명적인 상처가 된다. 여 러 학자의 연구를 살펴보면 온라인과 오프라인의 전혀 다른 환경 때문에 심리적으로나 현실적으로 소비자들이 경험하는 쇼핑 품질은 차이가 있다(Shin, Park, \& Kim, 2006; Moon, 2016; Hsieh, Chiu, \& Chang, 2018; Wong, Wong, \& Ke, 2018). 따라서 $\mathrm{O} 2 \mathrm{O}$ 기반 신선식품 전자상거래 시장의 활성화 를 위해서는 앞서 살펴본 문제에 관한 실무적 연구가 필요하다.

또한 기존 $\mathrm{O} 2 \mathrm{O}$ 및 신선식품의 선행연구를 살펴보면 많은 연구가 기술수용, 식품위생과 경영전략을 중심으로 이루어졌으 며(Won, Kang, \& Kim, 2017; Lee, Son, Ha, \& Kim, 2014; Yu, Yang, Sun, Qin, \& Xu, 2015; Lee \& Yang, 2017), 그러 나 빠르게 확대되고 있는 전자상거래 $\mathrm{O} 2 \mathrm{O}$ 기반 신선식품 시
장보다 관련 연구는 매우 부족하고, 특히 $\mathrm{O} 2 \mathrm{O}$ 기반 신선식품 앱의 품질 및 지속적인 사용의도에 대한 실증연구는 거의 없 는 실정이다.

그러므로 본 연구는 $\mathrm{O} 2 \mathrm{O}$ 기반 신선식품 전자상거래의 발 전을 촉진하기 위해 사용 경험 있는 소비자를 대상으로 $\mathrm{O} 2 \mathrm{O}$ 기반 신선식품 전자상거래가 지속적인 사용의도에 어떤 요인 이 영향을 미칠 것인지 규명하고 분석하고자 한다. 이러한 본 연구의 목적을 좀 더 구체적으로 제시하면 다음과 같다. 첫째, 기존의 $\mathrm{O} 2 \mathrm{O}$ 신선식품 전자상거래의 개념과 유형을 파악하고, 문헌연구를 통해 $\mathrm{O} 2 \mathrm{O}$ 기반 신선식품 전자상거래에 적합한 품 질요인을 찾아보고자 한다. 둘째, 기존의 D\&M 모델 선행연구 를 검토하고, $\mathrm{O} 2 \mathrm{O}$ 기반 신선식품 전자상거래 품질요인이 만 족도에 어떤 영향을 미치는지 확인하고자 한다. 셋째, UTAUT 모델과 ECM-ISC 모델의 선행연구를 파악하고 인지된 용이성, 인지된 유용성, 기대확신, 자기효능감, 사회적 영향 등 중요한 변수들을 도출하고자 한다. 도출한 변수는 $\mathrm{O} 2 \mathrm{O}$ 기반 신선식 품 전자상거래의 지속적 사용의도에 어떠한 영향을 미치는지 확인하고자 한다. 넷째, 실증분석 결과에 태도로 신선식품 전 자상거래 기업에 대한 지속적인 서비스의 개발 및 마케팅 전 략 등의 측면에서 효과적인 실무적 시사점을 도출하고자 한다.

\section{2. 이론적 배경 및 가설설정}

\section{1. $\mathrm{O} 2 \mathrm{O}$ 신선 농산품 전자상거래}

020 신선식품 전자상거래는 새로운 개념이기 때문에 아직 까지 명확하게 개념이 정립되지 않았다. 일반적으로 $\mathrm{O} 2 \mathrm{O}$ 신 선식품 전자상거래란 소비자들이 자신의 컴퓨터나 모바일 단 말기를 이용해서 식품을 주문하고 대금을 결제한 후, 오프라인 으로 식품을 제공받는 서비스이다. 즉 소비자가 온라인 가상점 포에서 상품을 주문하면, 오프라인 배송업체와의 협업을 통해 상품을 배송하고, 전자상거래 업체는 물류비용을 줄이는 효과 를 통해 가격이 비교적 낮더라도 더 많은 상품을 취급할 수 있다. $\mathrm{O} 2 \mathrm{O}$ 신선식품 전자상거래의 상품 배송은 전치창고를

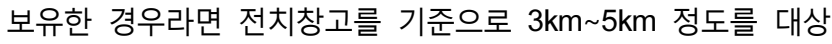
시장의 상권으로 설정하고, 상품 배송기간은 1 2시간 내로 배송서비스를 제공하기도 한다. 또한, $\mathrm{O} 2 \mathrm{O}$ 신선식품 전자상거 래는 직배송뿐만 아니라 오프라인 편의점이나 슈퍼마켓 업체 와 협력하는 방식, 또는 이를 플랫폼화하는 운영방식으로 존재 한다(iRecearch, 2018).

본 연구에서는 소비자가 앱을 통해 주문하고, 오프라인으로 식품을 제공받는 서비스에 초점을 맞추고 있으므로 $\mathrm{O} 2 \mathrm{O}$ 신선 식품 전자상거래는 소비자가 앱에서 신선식품을 주문하고, 배 송수단을 통해 주문자가 원하는 장소에서 신선식품을 즐길 수 있는 소비방식을 지칭한다. 중국 $\mathrm{O} 2 \mathrm{O}$ 신선식품 전자상거래 플랫폼은 공급망 전체에 관여하여 상품의 품질과 만족도를 높 일 수 있고, 현장에서 식재료에 대한 가공작업이 가능하므로 상품의 손실률을 줄일 수 있다. 특히 $\mathrm{O} 2 \mathrm{O}$ 신선식품 전자상거 래는 1 2시간 내 배송을 수행하여 빠른 배송을 원하는 소비 자를 유지하는 데 유리하다.

그러나 자본과 원가에 대한 투자를 많이 필요로 한다. 콜드 체인 배송망을 자가 구축해야 할 뿐만 아니라, 신선도를 유지 해야 하는 상품의 비율이 비교적 높아 수준 높은 관리를 필요 
로 한다. 특히 합작의 경우, 콜드체인 물류창고 관리를 합작업 체의 매장에 의지해야 하므로 상품 손실률이 높다. 또한, 수준 높은 수요예측과 재고 배치 능력이 요구된다. 가령 프로모션으 로 인해 특정 지역에서 수요가 급격히 증가할 경우, 합작 매장 의 창고가 수용하지 못할 수도 있다.

\section{2. 지속적 사용의도}

지속적 사용의도는 소비자들이 사용하거나 구매하기 사전의 행위로 실제 사용행동을 유도한다. Bhattacherjee(2001, 2008) 의 연구에서 지속적 사용의도를 소비자가 해당 시스템을 처음 시도해본 후 지속적으로 사용할 의도라고 정의하였다. 또한, 지속적 사용의도는 만족 이후의 후속 행동으로서, 제품이나 서 비스를 경험한 후 만족하여 직접 재사용하거나, 주변 사람에게 사용할 것을 추천하거나 같이 지속적 사용하려는 의도로 정의 할 수 있다(Hashim \& Tan, 2015).

$\mathrm{Gu}$ and Lee(2016)는 모바일 결제서비스 관점에서 기술수용 모형을 기반으로 모바일 결제 앱의 지속적 사용의도에 연구를 진행하였으며, 지속적 사용의도는 모바일 결제 앱에서 제공하 는 서비스를 향후에도 계속 사용할 의향 정도로 정의하였다.

Wang and $\operatorname{Kim}(2017)$ 는 업무-기술적합(TTF)모형과 기대확 신이론을 이용하여 $\mathrm{O} 2 \mathrm{O}$ 전자상거래 모바일 플랫폼에 대한 소 비자의 지속적인 사용의도에 관한 연구를 진행하였다. 연구결 과 만족도가 매우 중요한 요인으로 나타났지만, 만족한 느낌을 지속적인 사용의도로 전환시키는 것이 더 중요하다고 분석하 였다. Lee et al.(2018)은 모바일 O2O 커머스 지속이용의도에 영향을 미치는 전환 불안감에 대한 연구하였다. 연구결과 지속 적 사용의도는 실제 모바일 $\mathrm{O} 2 \mathrm{O}$ 커머스의 지속적 사용으로 이어지는 것을 확인하였다. 이외에도 많은 선행연구에서 지속 적 사용의도가 전자상거래의 성공 여부를 판단하는 중요한 변 수로 활용되어 왔어, 그러므로 본 연구에서는 지속적 사용의도 를 도입하였다.

\subsection{UTAUT Model 및 ECM-ISC Model}

기술수용이론은 소비자들이 신기술이나 서비스를 인지하고 사용하는 관계를 설명하거나 새로운 정보시스템이 초기 단계 에 정착될 수 있도록 하는데 많은 기여를 하였다(Davis, 1989). 소비자들이 처음에 이용했던 정보기술에 대해 어떤 수 용의지를 가지고 있는지를 분석하는 것이다. 특히 새로운 특정 정보기술을 도입하면 소비자가 자신의 업무 성과가 증가될 것 이라고 믿는 정도를 인지된 유용성으로, 신기술의 이용 방법에 큰 노력이 들지 않을 것이라고 믿는 정도를 인지된 사용용이 성으로 정의한 후, 인지된 유용성과 인지된 사용용이성이 신기 술에 대한 소비자들의 태도와 수용의지에 간접적 또는 직접적 으로 영향을 미침을 실증적인 분석하였다(Oghuma et al., 2016; Lin et al., 2017). 기술수용이론은 비록 지속적인 사용 의도를 설명하지는 못했지만, 이론적으로 사용자 인지에 근거 한 정보기술 사용을 예측하는 대표적인 모델이다. 그러나 소비 자 수용 전의 요인들이 소비자의 직접적인 경험을 통하여 시 스템 채택 후에 사용자의 신념이 변화할 수 있는 가능성과 그 에 따른 결과를 설명하지 못한다는 한계가 있다(Karahanna \& Straub, 1999; Bhattacherjee et al., 2008; Kim, 2010). 따라서 소비자들은 초기 정보시스템 수용 시점과 기술이나 정보 채택 이후 시스템의 사용에 대한 심리적 태도에 차이가 발생하게
된다.

한편, 기대확신모형은 소비자의 지속적 행위를 설명하기 위 해 소비자가 기대하는 확신 정도가 이용 후 인지된 유용성 그 리고 만족 수준에 미치는 영향력을 분석하고자 한 모형이다 (Bhattacherjee, 2001; Joo et al., 2018). 기대확신이론은 정보 기술 사용의도를 성과, 확신, 만족과 같은 지속적인 사용의도 와 별개로 사용전 기대 측정을 통해 설명하는 지속적인 사용 의도를 측정하는 모형이다. 또한 그 이론은 이용 후 채택되는 정보기술 이용의도는 구체적인 이용 후의 경험에 근거한 일시 적인 것인데 반해, 기술수용모형은 소비자의 사용경험이나 일 시적인 결과보다 우수한 인지된 유용성, 자세와 같은 더욱 일 반적인 인지를 포함하고 있다. 그러므로 기술수용이론 및 기대 확신이론을 결합한 모형은 소비자들이 정보시스템의 지속사용 의도를 더욱 잘 예측하고 설명을 할 수 있다. 또한, Bhattacherjee et al.(2008)은 기대확신모형에 TAM을 통합하는 과정에서 사용 후의 기대 대신 인지된 유용성을 사용했는데 UTAUT의 성과기대, 노력기대, 사회적 영향은 TAM의 인지된 유용성, 인지된 사용용이성 및 주관적 규범을 근간으로 설계되 었다. 그래서 성과기대, 노력기대 대신 인지된 유용성, 인지된 사용용이성을 차용하는데 큰 문제가 없을 것으로 판단된다.

Vedadi and Warkentin(2016)은 모바일 은행 앱의 지속적 사용의도에 대한 연구에서 Bhattacherjee(2001)의 기대확신모 형을 기반으로 연구를 위해 제안된 모형은 $67 \%$ 정도 높은 설 명력을 보였고, 전체 가설은 모두 채택되었다. Chen et al. (2018)의 모바일 $\mathrm{O} 2 \mathrm{O}$ 서비스 지속사용의도에 대한 연구에서는 기존의 기대확신모형을 이용하여 연구를 진행하였으며 기대확 신모형의 제시한 요인들이 모두 유의한 영향관계가 있음을 확 인하였다.

Joo et al.(2018)은 스포츠O2O서비스 앱을 이용한 소비자들 을 대상으로 한 연구에서 기대확신모형을 이용하여 헬스리터 러시(health literacy)와 혁신성을 추가하였으며, 헬스리터러시, 혁신성이 인지된 유용성에 미치는 유의한 영향을 확인하였다. Kang and Namkung(2018)은 O2O 식품 전자상거래를 이용하 는 한국 소비자를 대상으로 한 연구에서 기술수용모형의 인지 된 유용성, 인지된 사용용이성을 매개변수로 설정하고, 정보 품질과 정보의 확실성(source credibility)은 인지된 유용성, 인 지된 사용용이성에 유의한 영향을 미친다고 하였다.

Chung et al.(2014)은 모바일 증강현실 앱의 만족과 지속사 용을 설명하기 위해 UTAUT와 ECM을 통합한 연구모형을 설 정하여 UTAUT모형 변수인 성과기대, 노력기대를 이용하였으 며, 기대확신모형의 변수인 기대확신, 만족도, 지속적 사용의 도를 도입하고 분석하였다. 연구결과 노력기대가 만족도 및 지 속사용의도에 유의한 영향을 미치지 못하는 것을 확인하였다. 따라서 본 연구는 선행연구를 바탕으로 $\mathrm{O} 2 \mathrm{O}$ 신선식품 전자상 거래의 지속적 사용의도에 대한 검증을 위해 다음과 같은 가 설을 설정하였다.

$\mathrm{H} 1:$ 기대확신은 인지된 유용성에 정(+)의 영향을 미칠 것이 다.

H2: 기대확신은 만족도에 정(+)의 영향을 미칠 것이다.

H3: 자기효능감은 지속적 사용의도에 정(+)의 영향을 미칠 것이다.

H4: 인지된 유용성은 지속적 사용의도에 정(+)의 영향을 미 칠 것이다. 
H5: 인지된 이용용이성은 지속적 사용의도에 정(+)의 영향 을 미칠 것이다.

H6: 사회적 영향은 지속적 사용의도에 정(+)의 영향을 미칠 것이다.

H7: 만족도는 지속적 사용의도에 정(+)의 영향을 미칠 것이다.

\section{4. $\mathrm{O} 2 \mathrm{O}$ 신선 농산품 전자상거래의 품질요인}

$\mathrm{O} 2 \mathrm{O}$ 신선식품 전자상거래는 정보시스템을 기반으로 운영 되기 때문에 $\mathrm{O} 2 \mathrm{O}$ 신선식품 전자상거래 품질요인을 고려할 때 정보시스템의 품질요인 또한 고려되어야 한다. 정보시스템 품 질은 정보통신기술의 발전과 전자상거래 시스템 수요의 변화 에 따라 새로운 개념으로 제시되고 있다(DeLone \& McLean, 2004). 이와 더불어 정보시스템 성공에 영향을 미치는 정보시 스템 품질요인들에 대한 연구도 다양하게 진행되고 있다.

온라인 환경에서 제공하는 정보 및 서비스가 실제 상품을 제공하는 오프라인과 다를 경우 소비자는 실망감으로 인해 부 정적인 의도를 갖게 된다. 특히 $\mathrm{O} 2 \mathrm{O}$ 의 경우 구매와 동시에 제공된 서비스의 품질을 확인할 수 있으므로 소비자가 느끼는 상실감은 더 클 수밖에 없다. 그래서 다양한 정보시스템이나 전자상거래 품질요인에 관한 연구가 많이 이루어져 왔다.

Lee and Yang(2017)의 O2O 서비스의 품질이 사용자만족 과 재이용의도에 어떤 영향관계를 확인하기 위해 DeLone \& McLean(2004)의 정보시스템 성공모형을 기반으로 연구모형을 설정하였다. 연구결과는 $\mathrm{O} 2 \mathrm{O}$ 서비스의 시스템 품질이 소비자 만족도 및 재이용의도에 긍정적인 영향을 미치는 것으로 나타 났다.

Lee et al.(2018)은 DeLone and McLean(2004)이 제안한 정보시스템 성공모형에서 제시하고 있는 서비스 품질, 정보 품 질과 시스템 품질에 회사의 인지도 및 규모와 관련된 평판요 인을 추가하였으며 이들 요인이 O2O 서비스 재구매의도에 영 향을 미치는 품질요인이라고 제시하였다. 한편, 보안성이나 사 용자 인터페이스 등이 취약한 온라인 서비스의 경우 소비자 선택을 받을 수 없다. $\mathrm{O} 2 \mathrm{O}$ 의 온라인 및 오프라인 상호작용의 특성에 따라 오프라인 환경에서는 배송 품질을 매우 중요하게 고려해야 한다.

앞서 선행연구를 살펴보면 DeLone and McLean의 정보시 스템 성공모형은 전자상거래 시스템을 바탕으로 이루어지는 온라인 쇼핑몰, $\mathrm{O} 2 \mathrm{O}$ 서비스, 이러닝, 결제서비스 등 다양한 전 자상거래 분야에 적용되어왔다. 이에 본 연구에서는 기존의 DeLone and McLean(2004)의 서비스 품질, 시스템 품질과 정 보 품질이 소비자 만족도에 영향을 준다는 정보시스템 성공모 형에 오프라인 환경의 배송 품질을 추가하여 이 요인들이 만 족도에 어떠한 영향을 미치는가를 분석하고 검증하고자 한다. 따라서 본 연구는 선행연구를 바탕으로 $\mathrm{O} 2 \mathrm{O}$ 신선식품 전자상 거래의 품지요인이 만족도에 대한 검증을 위해 다음과 같은 가설을 설정하였다.

H8: 서비스 품질은 만족도에 정(+)의 영향을 미칠 것이다.

H9: 시스템 품질은 만족도에 정(+)의 영향을 미칠 것이다.

H10: 정보 품질은 만족도에 정(+)의 영향을 미칠 것이다.

H11: 배송 품질은 만족도에 정(+)의 영향을 미칠 것이다.

\section{5. 연구모형}

본 연구는 D\&M 모델, UTAUT 모델과 ECM-ISC 모델의 장 점을 통합하고 이들 모델 간의 관계를 분석하여 $\mathrm{O} 2 \mathrm{O}$ 기반 신 선식품 전자상거래의 지속적 사용의도에 관한 모델을 구축하 고자 하였다. 본 연구의 연구모형은 [Figure. 1]과 같다.

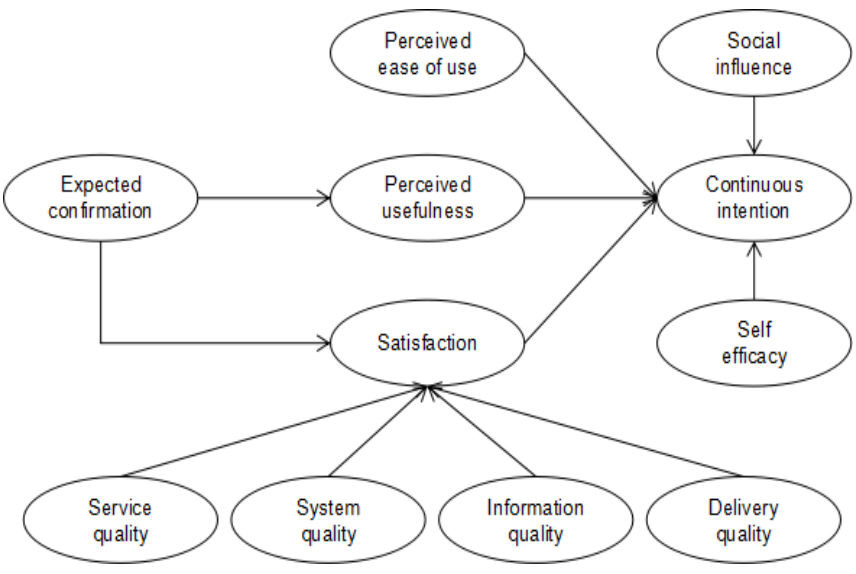

Figure 1: Research Model

\section{3. 연구방법}

\section{1. 자료수집 및 분석방법}

본 연구의 신뢰성과 타당성을 확보하기 위하여 본 조사를 실시하기 전에 전자상거래 분야의 교수, 박사 학위자 등 여러 전문가에게 측정 문항의 적합 여부를 검토하고 사전 조사를 통하여 설문지에서 잘못된 문제점들을 미리 찾아내어 수정함 으로써 본 조사를 보다 원활하고 효과적으로 수행할 수 있도 록 하였다. 이러한 과정을 거쳐 2018년 9월 18일부터 28일까 지 10 일 동안 사전조사를 온라인 방식으로 실시하였다. 총 30 부의 데이터를 회수하여 문제점 있는 설문문항을 보완하여 최 종 설문지를 제안하였다. 최종 본 조사는 $\mathrm{O} 2 \mathrm{O}$ 신선식품 전자 상거래 앱의 사용경험이 있는 중국소비자들을 대상으로 실시 하였으며, 2018년 10월 2일부터 11월 2일까지 온라인 리서치 사이트(問卷網, https://www.wenjuanwang.com)를 이용하여 설 문지 배포와 수집을 진행하였다. 본 연구를 통계적 분석을 위 해 IBM SPSS Statistics 25.0 및 IBM SPSS AMOS 25.0의 통 계프로그램을 사용하였다. 세부적으로는 첫째, 조사대상의 인 구통계학적 특성과 이용형태 특성 파악하기 위해 빈도분석을 실시하였다. 둘째, 타당성이 낮은 측정방법을 사용하는 경우 내적일관성을 확보할 수 없고, 내적일관성이 높다고 해석 타당 성이 반드시 높은 것도 아니다(Lee, 2017). 따라서 측정문항들 의 내적일관성 및 타당성 평가를 위해 신뢰성 분석, 탐색적 요 인분석 및 확인적 요인분석을 실시하였으며, 구성개념 간 타당 성을 평가하기 위해 판별타당분석을 실시하였다. 넷째, 본 연 구 목적에 해당하는 연구모형에 제시된 가설 검증을 위해 구 조방정식모형을 통해 구성개념 간 가설검증 및 모형의 적합도 를 검증하였다. 
Table 1: Operational Definitions

\begin{tabular}{|c|c|c|}
\hline Variable & Operation definition & Previous Studies \\
\hline Service Quality & $\begin{array}{l}\text { The extent to which services provided by } \mathrm{O} 2 \mathrm{O} \text { Fresh Agricultural } \\
\text { Products applications can satisfy consumer demand }\end{array}$ & $\begin{array}{l}\text { DeLone and McLean(2004), } \\
\text { Petter and McLean(2013), } \\
\text { Lee and Yang(2017) }\end{array}$ \\
\hline System Quality & $\begin{array}{l}\text { Consumers' cognition of system performance of O2O Fresh Agricultural } \\
\text { Products applications }\end{array}$ & $\begin{array}{l}\text { DeLone and McLean(2004), } \\
\text { Petter and McLean(2013), } \\
\text { Bahaddad(2017) }\end{array}$ \\
\hline $\begin{array}{l}\text { Information } \\
\text { Quality }\end{array}$ & $\begin{array}{l}\text { The extent to which information provided by } \mathrm{O} 2 \mathrm{O} \text { Fresh Agricultural } \\
\text { Products applications can satisfy consumer expectation and demand }\end{array}$ & $\begin{array}{l}\text { DeLone and McLean(2004), } \\
\text { Chung et al.(2016), } \\
\text { Wang et al.(2016) }\end{array}$ \\
\hline $\begin{array}{l}\text { Delivery } \\
\text { Quality }\end{array}$ & $\begin{array}{l}\text { Service provision level of } \mathrm{O} 2 \mathrm{O} \text { Fresh Agricultural Products applications } \\
\text { to quickly, exactly, and safely deliver the ordered products to } \\
\text { consumers within the limit of time. }\end{array}$ & $\begin{array}{l}\text { Kim et al.(2011), } \\
\text { Oh and Hyun(2016), } \\
\text { Dai and Lee(2018) }\end{array}$ \\
\hline $\begin{array}{l}\text { Perceived } \\
\text { Usefulness }\end{array}$ & $\begin{array}{l}\text { The degree of ease associated with the use of the } \mathrm{O} 2 \mathrm{O} \text { Fresh } \\
\text { Agricultural Products applications. }\end{array}$ & $\begin{array}{l}\text { Venkatesh et al.(2003), } \\
\text { Oghuma et al.(2016), } \\
\text { Lin et al.(2017), } \\
\text { Kang and Namkung(2018) }\end{array}$ \\
\hline $\begin{array}{l}\text { Perceived } \\
\text { Ease of use }\end{array}$ & $\begin{array}{l}\text { The degree to which an individual believes that using the } \mathrm{O} 2 \mathrm{O} \text { Fresh } \\
\text { agricultural products applications. }\end{array}$ & $\begin{array}{l}\text { Venkatesh et al.(2003), } \\
\text { Chung et al.(2014), } \\
\text { Kang and Namkung(2018) }\end{array}$ \\
\hline Social Influence & $\begin{array}{l}\text { Psychological response of consumers' use of } \mathrm{O} 2 \mathrm{O} \text { Fresh Agricultural } \\
\text { Products applications and influence of the surrounding environment }\end{array}$ & $\begin{array}{l}\text { Venkatesh et al.(2003), } \\
\text { Kim and Rha(2017), } \\
\text { Roh and Park(2018) }\end{array}$ \\
\hline $\begin{array}{l}\text { Expected } \\
\text { Confirmation }\end{array}$ & $\begin{array}{l}\text { Degree of consistency between use experience of } \mathrm{O} 2 \mathrm{O} \text { Fresh } \\
\text { Agricultural Products applications and consumer experience }\end{array}$ & $\begin{array}{l}\text { Bhattacherjee et al.(2008), } \\
\text { Lin et al.(2017), } \\
\text { Oghuma et al.(2016) }\end{array}$ \\
\hline Self-Efficacy & $\begin{array}{l}\text { The degree to which an individual beliefs the he or she has the ability } \\
\text { to perform specific task using O2O Fresh Agricultural Products } \\
\text { applications. }\end{array}$ & $\begin{array}{c}\text { Bhattacherjee et al.(2008) } \\
\text { Lin et al.(2017), } \\
\text { Gu and Lee(2017) }\end{array}$ \\
\hline Satisfaction & Consumer satisfaction of $\mathrm{O} 2 \mathrm{O}$ Fresh Agricultural Products applications & $\begin{array}{l}\text { Bhattacherjee et al.(2008), } \\
\text { Lin et al.(2017), } \\
\text { Oghuma et al.(2016) }\end{array}$ \\
\hline $\begin{array}{l}\text { Continuous } \\
\text { Intention }\end{array}$ & $\begin{array}{l}\text { Intention of consumers to continue using } \mathrm{O} 2 \mathrm{O} \text { Fresh Agricultural } \\
\text { Products applications }\end{array}$ & $\begin{array}{l}\text { Bhattacherjee et al.(2008), } \\
\text { Lin et al.(2017), } \\
\text { Oghuma et al.(2016) }\end{array}$ \\
\hline
\end{tabular}

\section{2. 번수의 조작적 정의}

본 연구에서는 $\mathrm{O} 2 \mathrm{O}$ 기반 신선식품 전자상거래 앱의 지속 적 사용의도에 미치는 영향을 알아보기 위해 기존의 선행연구 를 토대로 설문을 총 44문항으로 구성하였다(Table 1 참고). 조사대상의 인구통계학 특성은 총 5 개 문항으로 구성하였다. 인구통계학 특성을 제외한 측정변수들은 모두 Likert 5점 척도 에 의해 조사하였다. 설문조사는 사전조사와 본 조사로 구분하 여 진행하였다.

\section{4. 실증분석 및 가설검증}

\section{1. 표본의 특성}

본 연구에 설문지 대상의 인구사회학적 특성을 살펴보면, 성별의 경우 남자는 294 명(42.9\%), 여자는 391명(57.1\%)로 남 자와 여자의 비율 차이가 거의 없는 것으로 나타났다. 결혼여
부의 분포는 기혼은 220 명(32.1\%), 미혼은 465 명(67.9\%)로 미 혼이 기혼에 비해 많은 것으로 나타났다. 연령의 분포를 살펴 보면, 20 대(49.6\%) 및 30대(37.4\%)의 응답자가 본 설문조사에 참여하였다. 최종학력은 대학교 졸업 395 명 $(57.7 \%)$ 으로 차지 하였으며, 직업 부분은 회사원이 303명(44.2\%), 주부 152명 $(22.2 \%)$ 및 학생 150 명(21.9\%)으로 높은 비중을 차지하였다. 또한 응답자의 월 평균소득은 $5,001-8,000$ 위안이 302 명 (46.5\%)으로 가장 높은 비중으로 나타났다.

\section{2. 측정모형의 평가}

일반적으로 신뢰성의 측정방범은 재감사법, 반분법, 내적 일 관성을 구하여 측정하는 방법이 있다(Lee, 2017). George and Mallery(2016)는 사회과학분야에서 크론바하 알파계수 (Cronbach's alpha)가 0.8 이상이면 신뢰성이 양호한 것으로 판단하고 있다. 본 연구에서는 측정변수의 신뢰성을 분석한 결 과, 모든 변수의 크론바하 알파계수가 0.8 이상으로 높게 나타 나 측정변수들의 신뢰성은 양호한 것으로 판단되었다. 한편, 본 연구의 타당성을 검증하기 위해 확인적 요인분석을 실시하 
였다. 확인적 요인분석은 이미 탐색적으로 연구되었거나 또는 이론적인 근거에 의하여 측정구조모형이 가설적으로 구축된 것을 확인하기 위하여 실시하는 분석방법이다(Bae, 2017). 일 반적으로 연구모형에 대한 타당성은 주로 탐색적 요인분석을 이용하지만, 탐색적 요인분석을 실시한 후 확인적 요인분석을 통해 재검증한다면 보다 타당한 결과를 도출할 수 있다. 그러 므로 본 연구는 요인과 측정문항 간의 관계를 평가하기 위해 IBM AMOS Ver 25.0을 이용하여 확인적 요인분석을 실시하였
다. 분석결과에 따라 모든 측정요인들이 $\mathrm{SMC}$ (다중상관지수)은 0.4 이상, 합성신뢰도(Composite Reliability: $\mathrm{CR}$ )는 0.7 이상, 평균분산추출(Average Variance Extracted: AVE)은 0.5 이상으 로 나타나 모든 문항들이 통계적으로 유의함이 확인하였다. 측 정모형의 적합도(Model $\mathrm{Fit}$ )는 $\mathrm{CMIN} / \mathrm{DF}=1.886, \mathrm{GFI}=0.905$, AGFI=0.889, NFI=0.907, $\mathrm{CFI}=0.954, \quad \mathrm{IFI}=0.954, \quad \mathrm{RMSEA}=$ 0.037 로 나타나 측정모형은 적합하다고 판단하였다. 확인적 요 인분석의 결과는 <Table 2>과 같다.

Table 2: Convergent validity and reliability

\begin{tabular}{|c|c|c|c|c|c|c|}
\hline Construct & Item & Factor Loading & SMC & CR & AVE & Cronbach's $\alpha$ \\
\hline \multirow{4}{*}{$\begin{array}{c}\text { Expected } \\
\text { Confirmation }\end{array}$} & DC1 & 0.756 & 0.572 & \multirow{4}{*}{0.871} & \multirow{4}{*}{0.628} & \multirow{4}{*}{0.854} \\
\hline & DC2 & 0.833 & 0.694 & & & \\
\hline & DC3 & 0.767 & 0.588 & & & \\
\hline & DC4 & 0.812 & 0.660 & & & \\
\hline \multirow{4}{*}{$\begin{array}{l}\text { Perceived } \\
\text { Usefulness }\end{array}$} & PU1 & 0.830 & 0.689 & \multirow{4}{*}{0.858} & \multirow{4}{*}{0.603} & \multirow{4}{*}{0.861} \\
\hline & PU2 & 0.862 & 0.743 & & & \\
\hline & PU3 & 0.795 & 0.633 & & & \\
\hline & PU4 & 0.844 & 0.712 & & & \\
\hline \multirow{4}{*}{$\begin{array}{l}\text { Perceived } \\
\text { Ease of use }\end{array}$} & PE1 & 0.754 & 0.568 & \multirow{4}{*}{0.901} & \multirow{4}{*}{0.694} & \multirow{4}{*}{0.885} \\
\hline & PE2 & 0.779 & 0.607 & & & \\
\hline & PE3 & 0.808 & 0.653 & & & \\
\hline & PE4 & 0.763 & 0.581 & & & \\
\hline \multirow{4}{*}{ Satisfaction } & SAT1 & 0.687 & 0.471 & \multirow{4}{*}{0.888} & \multirow{4}{*}{0.667} & \multirow{4}{*}{0.812} \\
\hline & SAT2 & 0.686 & 0.47 & & & \\
\hline & SAT3 & 0.754 & 0.568 & & & \\
\hline & SAT4 & 0.793 & 0.629 & & & \\
\hline \multirow{4}{*}{ Self-Efficacy } & SE1 & 0.773 & 0.598 & \multirow{4}{*}{0.821} & \multirow{4}{*}{0.535} & \multirow{4}{*}{0.896} \\
\hline & SE2 & 0.771 & 0.594 & & & \\
\hline & SE3 & 0.801 & 0.642 & & & \\
\hline & SE4 & 0.775 & 0.601 & & & \\
\hline \multirow{4}{*}{ Social Influence } & SI1 & 0.875 & 0.766 & \multirow{4}{*}{0.903} & \multirow{4}{*}{0.701} & \\
\hline & $\mathrm{SI} 2$ & 0.864 & 0.747 & & & \\
\hline & $\mathrm{SI} 3$ & 0.767 & 0.588 & & & 0.902 \\
\hline & $\mathrm{SI} 4$ & 0.752 & 0.565 & & & \\
\hline & $\mathrm{Cl} 1$ & 0.849 & 0.720 & & & \\
\hline Continuous & $\mathrm{Cl} 2$ & 0.876 & 0.768 & & & \\
\hline Intention & $\mathrm{Cl} 3$ & 0.821 & 0.675 & 0.861 & 0.609 & 0.862 \\
\hline & $\mathrm{Cl} 4$ & 0.800 & 0.641 & & & \\
\hline & SQ1 & 0.838 & 0.702 & & & \\
\hline & SQ2 & 0.748 & 0.559 & & & \\
\hline Service Quality & SQ3 & 0.702 & 0.493 & 0.856 & 0.598 & $0.8 / 0$ \\
\hline & SQ4 & 0.799 & 0.638 & & & \\
\hline & PQ1 & 0.819 & 0.671 & & & \\
\hline & PQ2 & 0.938 & 0.879 & & & \\
\hline System Quality & PQ3 & 0.763 & 0.582 & 0.900 & 0.693 & 0.900 \\
\hline & PQ4 & 0.798 & 0.636 & & & \\
\hline & IQ1 & 0.775 & 0.600 & & & \\
\hline & IQ2 & 0.858 & 0.736 & & & \\
\hline Information Quality & IQ3 & 0.736 & 0.541 & 0.857 & 0.601 & 0.857 \\
\hline & IQ4 & 0.725 & 0.526 & & & \\
\hline & LQ1 & 0.867 & 0.751 & & & \\
\hline & LQ2 & 0.704 & 0.496 & & & \\
\hline Delivery Quality & LQ3 & 0.640 & 0.410 & 0.823 & 0.542 & 0.819 \\
\hline & LQ4 & 0.713 & 0.509 & & & \\
\hline
\end{tabular}


또한 본 연구의 판별타당성분석 결과는 <Table 3>에 따라 면 잠재변수 간 상관계수 중에서 가장 큰 값은 0.662 이다. 상 관계수의 제곱값은 $0.438(0.662 \times 0.662)$ 이다. 측정변수의 평균 분산추출값은 상관계수의 제곱값보다 크므로 나타나 판별타당 성을 확보할 수 있다고 판단하였다.

\section{3. 가설검증}

일반적으로 사회과학 연구에서는 $\mathrm{a}=0.05$ (95\% 신뢰수준)를 기준하기 때문에, $\mathrm{p}$ 값이 0.05 보다 작으면 무조건 가설은 채택 되고, t값(C.R.)이 \pm 1.96 보다 클 때 연구가설이 채택된다(Lee, 2017). AMOS에서는 Regression Weights의 C.R.값으로 연구 가설의 채택여부를 판단한다. 또한 구조모형의 적합도(Model Fit)는 $\mathrm{CMIN} / \mathrm{DF}=2.002, \mathrm{GFI}=0.898, \mathrm{AGFI}=0.883, \mathrm{NFI}=0.899$, $\mathrm{CFI}=0.946, \mathrm{IFI}=0.947, \mathrm{TLI}=0.941, \mathrm{RMSEA}=0.039$ 로 양호한 것 으로 판단되었다. 본 연구의 가설검증 결과는 다음의 <Table 4>에 제시하였다.

\section{5. 결론}

\section{1. 요약 및 시사점}

본 연구에서는 기존의 $\mathrm{O} 2 \mathrm{O}$ 기반 신선식품 전자상거래에 대한 선행연구와 UTAUT모형, 확장된 기대확신모형에 대한 선 행연구를 검토하였으며 또한, 정보시스템 성공모형을 참고로 하여 $\mathrm{O} 2 \mathrm{O}$ 기반 신선식품 전자상거래의 만족도에 영향을 미치 는 품질요인(서비스 품질, 시스템 품질, 정보 품질, 배송 품질) 을 규명하였으며, 규명된 요인들을 재정의하였다. 이러한 요인 들이 $\mathrm{O} 2 \mathrm{O}$ 기반 신선식품 전자상거래의 지속적 사용의도에 미 치는 영향에 대해 검증함으로써 신선식품 전자상거래 기업에 대한 지속적인 서비스의 개발 및 마케팅 전략 등의 측면에서 효과적인 시사점을 제시하고자 하였다. 본 연구의 목적을 확보 하기 위해 가설의 검증결과를 정리하면 다음과 같다.

우선, UTAUT모형, 확장된 기대확신모형에 대한 가설의 검 증결과는 다음과 같다. 기대확신이 인지된 유용성 및 만족도에 정(+)의 영향을 미치는 것으로 나타나 가설1, 가설2는 채택되 었다. 특히 기대확신은 인지된 유용성에 더욱 큰 영향을 미치

Table 3: Discriminant validity

\begin{tabular}{|c|c|c|c|c|c|c|c|c|c|c|c|}
\hline Construct & DC & PU & PE & SAT & SE & SI & $\mathrm{Cl}$ & $S Q$ & $P Q$ & IQ & $D Q$ \\
\hline $\mathrm{DC}$ & .628 & & & & & & & & & & \\
\hline $\mathrm{PU}$ & .498 & .603 & & & & & & & & & \\
\hline $\mathrm{PE}$ & .511 & .567 & .694 & & & & & & & & \\
\hline SAT & .373 & .232 & .333 & .667 & & & & & & & \\
\hline SE & .529 & .283 & .441 & .035 & .535 & & & & & & \\
\hline $\mathrm{SI}$ & .211 & .121 & .182 & .192 & .184 & .701 & & & & & \\
\hline $\mathrm{Cl}$ & .501 & .483 & .572 & .387 & .374 & .467 & .609 & & & & \\
\hline$S Q$ & .186 & .049 & .147 & -.013 & .662 & .169 & .017 & .598 & & & \\
\hline$P Q$ & .012 & -.047 & .024 & .051 & .092 & .004 & -.008 & .104 & .693 & & \\
\hline IQ & .174 & .209 & .215 & .041 & .412 & .192 & .168 & .322 & .069 & .601 & \\
\hline $\mathrm{DQ}$ & .250 & .272 & .306 & .085 & .460 & .298 & .138 & .284 & -.007 & .377 & .542 \\
\hline
\end{tabular}

Note: The square root of AVE (italic at diagonal).

Table 4: Influence paths and hypotheses results.

\begin{tabular}{|c|c|c|c|c|}
\hline Hypotheses & Estimate & S.E. & t-value(P-Value) & Result \\
\hline H1: Expected Confirmation $\rightarrow$ Perceived Usefulness & 0.556 & 0.044 & $12.603\left(^{* * *}\right)$ & Accepted \\
\hline H2: Expected Confirmation $\rightarrow$ Satisfaction & 0.185 & 0.033 & $5.615\left(^{* * *}\right)$ & Accepted \\
\hline H3: Self-Efficacy $\rightarrow$ Continuous Intention & 0.259 & 0.047 & $5.532\left({ }^{* * *}\right)$ & Accepted \\
\hline H4: Perceived Usefulness $\rightarrow$ Continuous Intention & 0.326 & 0.044 & $7.390($ ***) & Accepted \\
\hline H5: Perceived Ease of use $\rightarrow$ Continuous Intention & 0.276 & 0.051 & $5.407(* \star)$ & Accepted \\
\hline H6: Social Influence $\rightarrow$ Continuous Intention & 0.296 & 0.038 & $7.748(* *)$ & Accepted \\
\hline H7: Satisfaction $\rightarrow$ Continuous Intention & 0.261 & 0.049 & $5.333(* * *)$ & Accepted \\
\hline H8: Service Quality $\rightarrow$ Satisfaction & 0.434 & 0.037 & $11.716\left(^{* \star *}\right)$ & Accepted \\
\hline H9: System Quality $\rightarrow$ Satisfaction & 0.024 & 0.030 & $0.788(0.431)$ & Rejected \\
\hline H10: Information Quality $\rightarrow$ Satisfaction & 0.122 & 0.041 & $3.009(0.003)$ & Accepted \\
\hline H11: Delivery Quality $\rightarrow$ Satisfaction & 0.227 & 0.045 & $5.032\left({ }^{* * *}\right)$ & Accepted \\
\hline
\end{tabular}


는 것으로 나타났다. 즉, 소비자의 사전 기대와 실제 사용성과 가 일치할수록 $\mathrm{O} 2 \mathrm{O}$ 신선식품 전자상거래 앱 소비자 자신의 목적에 유용하다고 인지하는 정도와 만족도가 높아진다. 자기 효능감이 지속적 사용의도에 정(+)의 영향을 미치는 것으로 나 타나 가설3은 채택되었다. 즉, 소비자들이 $\mathrm{O} 2 \mathrm{O}$ 기반 신선식품 전자상거래 앱의 사용에 대한 자신감이 높다고 생각하면, 서비 스를 비교적으로 쉽게 받아들인다고 해석할 수 있다.

인지된 유용성 및 인지된 사용용이성이 지속적 사용의도에 정(+)의 영향을 미치는 것으로 나타나 가설4, 가설5는 채택되 었다. 즉, 소비자들이 $\mathrm{O} 2 \mathrm{O}$ 기반 신선식품 전자상거래 앱의 사 용할 때, 자신의 목적에 유용하다고 느낄 수 있어야 지속적 사 용의도가 높아진다고 해석할 수 있다. 또한, $\mathrm{O} 2 \mathrm{O}$ 기반 신선식 품 전자상거래 앱의 사용방법이 쉽다고 인식할수록 자신의 목 적을 달성할 수 있으며 지속적 사용의도가 커진다는 것을 확 인하였다.

사회적 영향이 지속적 사용의도에 정(+)의 영향을 미치는 것으로 나타나 가설6은 채택되었다. 이는 $\mathrm{O} 2 \mathrm{O}$ 기반 신선식품 전자상거래 앱을 사용할 때 소비자들이 개인의 심리적인 요인 뿐만 아니라 외부 환경적인 요인이 타인과 주변 환경의 긍정 적인 영향을 받으면 지속적 사용의도를 향상시키는 데 도움을 줄 수 있다는 것이다. 만족도가 지속적 사용의도에 정(+)의 영 향을 미치는 것으로 나타나 가설7은 채택되었다. 지속적 사용 의도는 기업 관점에서 마케팅 비용 대비 큰 효율로 여겨진다. $\mathrm{O} 2 \mathrm{O}$ 기반 신선식품 전자상거래 앱에 만족한 소비자가 지속적 으로 사용하려는 의도가 더 크므로 기업은 소비자들이 만족도 를 높일 수 있는 방안에 더욱 집중하여 서비스를 제공하는 것 이 바람직하다.

둘째, 확장된 D\&M 모형의 품질요인에 대한 가설의 검증결 과는 다음과 같다. 서비스 품질이 만족도에 정(+)의 영향을 미 치는 것으로 나타나 가설8은 채택되었다. 즉 서비스의 품질이 좋으면 $\mathrm{O} 2 \mathrm{O}$ 기반 신선식품 전자상거래 앱의 제공자와 소비자 간에 긍정적인 인식이 형성되고, 또한, 소비자가 앱의 사용과 정에서 의견이나 문제 해결의 시간이 짧을수록 소비자들은 만 족스러운 것으로 볼 수 있다.

시스템 품질이 만족도에 정(+)의 영향을 미치는 것으로 나 타났으나 통계적으로 유의하지 않아서 가설9는 기각되었다. 이러한 결과는 선행연구 중 일부의 결과와 일치된다(정성립 외, 2013). 오늘날 전자상거래 기술이 발전하고 웹 사이트 또 는 모바일 소프트웨어 시스템은 과거에 비해 매우 안정적이고, 오류가 많이 발생하지 않는 상태이다. 또한, 현실적으로 $\mathrm{O} 2 \mathrm{O}$ 기반 신선식품 전자상거래 앱은 젊은 소비자들이 많이 사용하 고 있어 앱을 능숙하게 사용할 수 있으므로 시스템 품질이 좋 아져도 만족감을 충분히 느끼지 못한다고도 볼 수 있다.

정보 품질이 만족도에 정(+)의 영향을 미치는 것으로 나타 나 가설10은 채택되었다. 이러한 결과는 기존의 선행연구에서 나타난 분석 결과와 유사한 결과라 할 수 있다(이은용, 2016). 정보시스템에서는 소비자에게 보여줄 상품 정보의 품질이 매 우 중요하다. 만약 $\mathrm{O} 2 \mathrm{O}$ 기반 신선식품 전자상거래 앱에서 제 공한 정보와 실제 상품의 정보가 일치하지 않는다면 소비자들 이 만족감을 느끼지 못하게 되고, 특히 온라인 환경에서는 비 대면 거래라는 한계점이 있기 때문에 정확한 정보제공은 매우 중요하다.

배송 품질이 만족도에 정(+)의 영향을 미치는 것으로 나타 나 가설11은 채택되었다. 이는 배송 품질은 제시간에 맞춰 소 비자에게 주문한 상품을 안전하고 정확하게 배송해야 한다는
것이다. 배송 서비스는 온라인 쇼핑의 마지막 단계 임에도 불 구하고 그것의 품질이 소비자의 구매 만족도에 매우 큰 영향 을 주고 있다. 따라서 배송 품질이 좋아지면 소비자들의 만족 도가 향상될 수 있다.

한편, 현재 $\mathrm{O} 2 \mathrm{O}$ 신선식품 전자상거래의 사용이 점차 빠르 게 증가하고 있는 추세이다. 본 연구에서는 $\mathrm{O} 2 \mathrm{O}$ 기반 신선식 품 전자상거래의 지속적 사용모델을 구축하고자 하였다. 구체 적으로 본 연구의 실무적 시사점은 다음과 같다.

첫째, 본 연구에서는 중국 $\mathrm{O} 2 \mathrm{O}$ 기반 신선식품 전자상거래 앱에서 온라인·오프라인의 품질요인 향상이 소비자 만족도에 주요한 영향을 미칠 수 있음을 실증적으로 입증하였으며, 구체 적으로는 $\mathrm{O} 2 \mathrm{O}$ 기반 신선식품 전자상거래 앱의 서비스 품질, 시스템 품질, 정보 품질, 배송 품질에 따라 만족도의 중요성을 강조하고, 중국 $\mathrm{O} 2 \mathrm{O}$ 기반 신선식품 전자상거래 앱의 품질요 인 개선에 있어서 어떠한 차원에 중점을 두어야 할 것인가에 대해 실무적인 시사점을 제시하였다.

기업들은 빅 데이터를 통해 소비자들의 구매상품, 구매빈도 를 분석하여, 선호도에 따라 소비자가 주로 구매하는 시간대에 맞춰 앱을 통해 관련 상품 정보를 소비자에게 전송한다. 또는 배송속도를 높이기 위해 배송직원은 어느 한 창고에 고정되지 않고 소비자가 주문하면, 인공지능 시스템이 소비자, 창고 고 리고 배송지원 3 자의 위치를 분석하여 가장 가까운 위치에 있 는 배송직원 및 창고에서 상품을 출고한다. 앱의 예약배송서비 스를 제공하면서 아파트 단지 주변에 자체 운영하는 무인냉장 택배함도 설치할 수도 있다. 소비자들이 스마트폰 앱을 통해 $\mathrm{QR}$ 코드를 스캔해서 무인냉장 택배함에 보관한 신선식품을 출 고할 수 있다. 이렇게 하면 소비자 자신의 프라이버시를 보장 해줄 뿐만 아니라, 직원들의 시간과 노선을 미리 계획할 수 있 기 때문에 소비자들의 불필요한 기다림을 줄일 수 있다.

둘째, 본 연구에서는 기대확신이 인지된 유용성 및 만족에 유의한 영향을 미치는 것으로 나타났다. $\mathrm{O} 2 \mathrm{O}$ 신선식품 전자 상거래의 소비자가 사전 기대와 실제 성과를 비교하여 일치성 이 높을수록 자신이 사용했던 정보에 대해 만족하는 것으로 이해할 수 있다. 또한, 의사결정 유용성은 경험 후 형성된 소 비자의 사후 신념으로서, 기대와 성과가 일치할수록 특정 쇼핑 몰에서의 구매의사결정이 유용하다고 지각한다는 것을 알 수 있다. 따라서 $\mathrm{O} 2 \mathrm{O}$ 신선식품 전자상거래 기업들은 소비자의 사전 기대 요인을 충분히 고려하여야 할 것이다.

개인이 기대하는 요소는 주관성이 너무 강하고, 동시에 사 람마다 기대수준도 차이가 있다. 특히, 신선식품에 대한 개인 의 기대는 매우 큰 차이가 있다. 그러므로 기업들은 신선식품 표준화 체계를 수립하는 것이 매우 중요하며 소비자가 신선식 품을 구매하기 전에 기업은 앱을 통해 자체의 신선식품 표준 화 평가수준에 따른 관련 정보를 소비자에게 미리 제공하는 전략이 필요하다. 예를 들면 신선식품의 원산지, 친환경 식품, 식품의 맛, 크기 등의 정보를 제공해야 한다. 사전에 소비자에 게 제품의 표준화를 공지함으로써 소비자들로 하여금 소비과 정 중에 생기는 기대 불일치로 인한 불만족을 감소시킬 수 있 다.

셋째, 사회적 영향이 $\mathrm{O} 2 \mathrm{O}$ 신선식품 전자상거래 앱의 지속 적 사용의도 향상에 긍정적인 영향을 미치는 것으로 나타났다. 신선식품 전자상거래 기업들은 적극적인 소셜 미디어, 사회 홍 보 등을 통해 신선식품 전자상거래의 사회적 중요성을 강조해 야 한다. 예를 들면 기업들은 소셜 미디어와 소셜 네트워크를 통해 앱과 서비스를 홍보하거나 대중매체에 공익적인 광고를 
적극적으로 활용해야 한다. 최근 소비자들은 사회 공익성에 대 한 인식이 강화되어 기업의 광고가 공익성 위주로 구현될 때 기업의 이미지 제고에 도움이 된다. 본 연구결과에 따르면 $\mathrm{O} 2 \mathrm{O}$ 신선식품의 주요 사용 계층은 20,30 대이며, 이러한 소 비자들은 1 인가구처럼 독립적으로 생활하고 요리에 대한 지식 수준이 높은 편이라고 할 수 있다. 따라서 $\mathrm{O} 2 \mathrm{O}$ 신선식품 전 자상거래 기업들은 정기적으로 스마트폰 앱을 통해 목표 소비 자에게 요리법을 전달하는 등의 전략을 통해 소비자의 적극적 인 참여를 유도하는 동시에 소비자와 기업 간의 상호교류를 촉진할 수도 있다. 또한, 소비자 개인별 차이를 고려하여 앱의 사용법을 편리하고, 간단하게 설계할 필요가 있다. 이를 통해 앱의 학습 및 이용에 대한 소비자의 우려를 줄일 수 있으며 소비자들은 $\mathrm{O} 2 \mathrm{O}$ 신선식품 전자상거래 앱의 이용에 자신감을 가질 수 있을 것이다.

\section{2. 한계점 및 연구방향}

본 연구는 $\mathrm{O} 2 \mathrm{O}$ 기반의 신선식품 전자상거래 앱을 중심으 로 소비자의 지속적 사용의도에 영향을 미치는 요인에 관한 연구를 수행하고, 다양한 시사점을 도출하고자 하였으나, 다음 과 같은 일부 한계점이 있다. 첫째, 본 연구에서 표본의 크기 는 충분하였지만 대부분 설문지 참여자가 20 대, 30 대의 젊은 사람이기 때문에 연령의 경계를 넘어서는 일반화에는 일부 한 계가 있다고 할 수 있다. 또한, 온라인 설문조사의 구조적인 한계로 인해 응답자들의 측정 문항에 대한 이해정도에 따른 오류가 발생할 수 있다. 따라서 향후 연구에서 다양한 사회계 층의 표본을 대상으로 하는 연구와 더불어 온라인과 오프라인 의 조사방식을 병행할 필요도 있다고 판단된다.

둘째, 본 연구에서는 종속변수로써 $\mathrm{O} 2 \mathrm{O}$ 기반 신선식품 전 자상거래를 사용한 경험이 있는 소비자의 지속적 사용의도만 을 고려하고자 하였다. $\mathrm{O} 2 \mathrm{O}$ 기반 신선식품 전자상거래에서의 소비자 만족의 결과변수로서 지속적 사용의도 외에도 몰입이 나 신뢰도 등 다양한 변수들을 고려할 수 있을 것이다. 따라서 향후 연구는 이러한 점에 대한 추가적인 개선이 필요하다고 판단된다.

\section{References}

Bae, B. R. (2017). Amos 24 structural equation modeling, Crbooks.

Bahaddad, A. A. (2017). Evaluating M-commerce systems success: Measurement and validation of the DeLone and McLean model of IS success in Arabic society (GCC Case Study). Journal of Business Theory and Practice, 5(3), 156.

Bhattacherjee, A., Perols, J., \& Sanford, C. (2008). Information technology continuance: A theoretic extension and empirical test. Journal of Computer Information Systems, 49(1), 17-26.

Chen, L. Y., Klièek, T., \& Tsai, C. C. (2018). Exploring M2O Service System and Its Impact on Continuous Use Intention. International Journal of Advanced Studies in Computers, Science and Engineering, 75), 11-16.
Chung, N. H., Lee, H. A., \& Kim, J. M. (2014). Factors Affecting Satisfaction and Continued Usage Intention of Mobile Augmented Reality App on Cultural Heritage Tourism. Journal of Tourism and Leisure Research, 26(8), 55-72.

Dai, W. Q., \& Lee, J. H. (2018). Effects of Website Characteristics and Delivery Service Quality on Repurchase Intention. The International Journal of Industrial Distribution \& Business, 9(5), 17-24.

Delone, W. H., \& Mclean, E. R. (2004). Measuring e-commerce success: Applying the DeLone \& McLean information systems success model. International Journal of electronic commerce, 9(1), 31-47.

Gao, T. L., \& Ko, H. J. (2017). A Study on the Last Mile Delivery for B2C Fulfillment of Fresh Food e-Commerce in China. E-Trade Review, 15(2), 51-71.

George, D., \& Mallery, P. (2016). IBM SPSS statistics 23 step by step: $A$ simple guide and reference. Abingdon, United Kingdom: Routledge.

Gu, W., \& Lee, J. H. (2017). A Study on the Use Intention of Smart Home APP based on loT -Focusing on the China Market. The e-business studies, 18(6), 195-212.

Hashim, K. F., \& Tan, F. B. (2015). The mediating role of trust and commitment on members' continuous knowledge sharing intention: A commitment-trust theory perspective. International Journal of Information Management, 35(2), 145-151.

Hsieh, Y. H., Chiu, H. H., \& Chang, W. L. (2018). Examining the Determinants of Valuable Customer Experiences in $\mathrm{O} 2 \mathrm{O}$ Commerce Contexts. AMCIS2018.

iResearch(2018). 2018 China Fresh E-commerce Industry Consumption Report in 2018.

Joo, H. C., Kim, J. H., \& Kwon, H. I. (2018). An Intention of Consistent Use of Sport O2O Service App Based on Expectation-Confirmation Model. Korean journal of physical education, 57(2), 195-212.

Kang, J. W., \& Namkung, Y. (2018). The information quality and source credibility matter in customers' evaluation toward food $\mathrm{O} 2 \mathrm{O}$ commerce. International Journal of Hospitality Management. In Press, Corrected Proof

Karahanna, E., \& Straub, D. W. (1999). The psychological origins of perceived usefulness and ease-of-use. Information \& Management, 35(4), 237-250.

Kim, B. (2010). An empirical investigation of mobile data service continuance: Incorporating the theory of planned behavior into the expectation-confirmation model. Expert systems with Applications, 37(10), 7033-7039.

Kim, H. J. \& Rha, J. Y. (2017). Impacts of the O2O Mobile Order and Pay Services Continued Use Intention: Usage Frequency Moderating Effect. Journal of Consumption Culture, 20, 199-226.

Kim, J. H., Seo, S. S., \& Lee, J. H. (2011). A Study on the Influence of Customer Satisfaction and Loyalty by the Logistic Service Quality of Internet Shopping Mall. 
The Journal of Business Education, 26, 443-461.

Lee, H. Y. (2017). Research methodology, Crbooks.

Lee, K. R., Kim, M. S., \& Lee, S. J. (2018). A Study on the Influence of Telephone Apprehension Affecting Continuous Use Intention of Mobile O2O Commerce. Journal of Digital Contents Society, 19(4), 661-671.

Lee, O. J., \& Yang, D. W. (2017). A Study on the Effect of $\mathrm{O} 2 \mathrm{O}$ Service Quality on User Satisfaction and Intention of Reuse. Journal of Digital Convergence, 15(6), 165-178.

Lee, S. L., Son, J. Y., Ha, H. R., \& Kim, M. J. (2014). Consumer Anxiety on Foods hazards and Satisfaction with Food-related Life - Focusing on the effects of subjective consumer competency and safe food purchasing behavior -. Journal of consumer policy studies, 45(2), 137-157.

Lee, Y. M., Jun, S. Y., \& Choi, J. Y. (2018). A Comparative Study on Quality Factors Affecting Repurchase Intention of $\mathrm{O} 2 \mathrm{O}$ Service. The journal of professional management, 21(2), 403-427.

Lin, X., Featherman, M., \& Sarker, S. (2017). Understanding factors affecting users' social networking site continuance: A gender difference perspective. Information \& Management, 54(3), 383-395.

Mi, Y. C., Chang, K. J., \& Kyeong, S. H. (2017). A Study on the Adoption Intention of O2O-based Food Delivery Agency Service: Focused on the Small Traders in the Restaurant Industry. Korea Journal of Business Administration, 307), 1257-1282.

Moon, Y. J. (2016). The Study of Service Quality Model in O2O(Online-to-Offline) Context. Management \& Information Systems Review, 35(3), 213-230.

Oghuma, A. P., Libaque-Saenz, C. F., Wong, S. F., \& Chang, Y. (2016). An expectation-confirmation model of continuance intention to use mobile instant messaging. Telematics and Informatics, 33(1), 34-47.

Oh, M. G., \& Hyun, B. E. (2016). A Study on Logistics Service Quality and Repurchase Intention in the Mobile Food-Shopping. Korea Logistics Review, 26(4), 39-54.

Petter, S., DeLone, W., \& McLean, E. R. (2013).
Information systems success: The quest for the independent variables. Journal of management information systems, 29(4), 7-62.

Roh, M., \& Park, K. (2018). Adoption of O2O food delivery services in South Korea: The moderating role of moral obligation in meal preparation. International Journal of Information Management. In Press, Corrected Proof

Shin, J. Y., Park, S. Y., \& Kim, D. H. (2006). The Study on the Determinants of Online Shopping Purchase Intention: The Roles of Off-line Shopping Attributes. Korea Journal of Business Administration, 19(2), 529-560.

Vedadi, A., \& Warkentin, M. (2016). Continuance Intention on Using Mobile Banking Applications: A Replication Study of Information Systems Continuance Model. A/S Transactions on Replication Research, 2(1), 7.

Venkatesh, V., Morris, M. G., Davis, G. B., \& Davis, F. D. (2003). User acceptance of information technology: Toward a unified view. MIS quarterly, 425-478.

Wang, L. Y., \& Kim, M. J. (2017). A Study on the Customer Continuance Intention of $\mathrm{O} 2 \mathrm{O}$ E-commerce Mobile Platform. The e-business studies, 18(3), 187-199.

Won, J. Y., Kang, H. C., \& Kim, B. Y. (2017). The Effect of Food Online-to-Offline (O2O) Service Characteristics on Customer Beliefs using the Technology Acceptance Model. Culinary Science \& Hospitality Research, 23(7), 97-111.

Wong, R. M. M., Wong, S. C., \& Ke, G. N. (2018). Exploring online and offline shopping motivational values in Malaysia. Asia Pacific Journal of Marketing and Logistics, 30(2), 352-379.

Yu, B. H., Yang, Y. Q., Sun, J. L., Qin, J., \& Xu, Y. (2015). The Effects of Customer Offline Experience and Merchant Offline Presence on Customer Acceptance of Community $\mathrm{O} 2 \mathrm{O}$ Ecommerce. Chinese Journal of Management, 12(11), 1658-1663.

Yu, X., \& Ren, X. (2018). The impact of food quality information services on food supply chain pricing decisions and coordination mechanisms based on the o2o e-commerce mode. Journal of Food Quality, 2018(3), 1-18. 